\title{
PHENETHYL ALCOHOL IS AN EFFECTIVE NON-TRADITIONAL PRESERVATIVE AGENT FOR COSMETIC PREPARATIONS
}

\section{SASITHORN SIRILUN ${ }^{1}$, CHAIYAVAT CHAIYASUT ${ }^{1}$, BHAGAVATHI SUNDARAM SIVAMARUTHI ${ }^{1}$, SARTJIN PEERAJAN ${ }^{2}$ NAPHATSORN KUMAR ${ }^{3}$, PERIYANAINA KESIKA ${ }^{1 *}$}

${ }^{1}$ Department of Pharmaceutical Sciences, Faculty of Pharmacy, Chiang Mai University, Chiang Mai 50200, Thailand. ${ }^{2}$ Health Innovation Institute, Chiang Mai 50200, Thailand. ${ }^{3}$ School of Cosmetic Science, Mae Fah Luang University, Chiang Rai 57100, Thailand. Email: p.kesika@gmail.com; kesika.p@cmu.ac.th

Received: 19 March 2017, Revised and Accepted: 26 April 2017

\begin{abstract}
Objective: Preservatives are used in the cosmetic products to protect the potential growth of microbes, therefore, to prolong the shelf life of products, and to protect the consumer from infections. However, several preservatives can cause various health problems, and the safety profiles of those preservatives are still unclear. Many natural substances are used in the cosmetic products to substitute the traditional preservatives. The present study deals with the evaluation of conservative nature of phenethyl alcohol (PEA) in three cosmetic formulations (emulsion, cleansing, and conditioner).
\end{abstract}

Methods: Three different concentrations of PEA $(0.3 \%, 1 \%$, and $2.5 \%)$ were used in cosmetic formulations. The physical appearance of the formulas was assessed manually, and the antimicrobial nature of PEA and PEA-containing cosmetic formulations was evaluated by agar well plate assay.

Results: The use of PEA has not affected the physical appearance and quality of the formulations, except the high concentration of PEA in the cleansing solution, which reduced the foam formation. The minimal required concentration of PEA in emulsions and cleansings was $1.0 \%$ and $2.5 \%$ in the conditioners. All cosmetic preparations were subjected to antibacterial and antifungal evaluation. The alkaline pH ( $>8)$ affected the antimicrobial activity of PEA in a cosmetic product.

Conclusion: The results suggested that PEA is a potent non-traditional preservative for the cosmetic formulations, especially, in the emulsion, cleansing solutions, and conditioners.

Keywords: Cosmetic, Phenethyl alcohol, Preservative, Self-preserving cosmetic.

(C) 2017 The Authors. Published by Innovare Academic Sciences Pvt Ltd. This is an open access article under the CC BY license (http://creativecommons. org/licenses/by/4. 0/) DOI: http://dx.doi.org/10.22159/ajpcr.2017.v10i8.18572

\section{INTRODUCTION}

Preservatives are used in the cosmetic preparations to prevent microbial growth in the product for prolonging the shelf life and protecting the consumer from infection [1]. The combination of various preservatives has been recommended to the cosmetic industries to obtain the adequate protection from microbial contamination [2]. However, safety of these compounds is still unclear, for example, parabens, the most commonly used preservatives, show weak estrogen-like activity and may lead to breast cancer [3]. Similarly, the potential skin irritation among the consumers of formaldehyde-releasing compounds (such as imidazolidinyl urea) containing products has been reported [1]. Even some of the cosmetic manufacturers restrict the use of few permitted preservatives in their products and advertising the products such as parabens free, chlorine free, iodine free, formaldehyde free, and glycol free [4]. Nowadays, several cosmetic industries are concentrating more on preservative-free products, which referred that the preparation of cosmetic product without addition of any of the substance that exerts antimicrobial activity. However, preservative-free products in market usually contain substances with antimicrobial activity, these compounds were not yet identified as the preservatives by the European Scientific Committee and were not indicated in Annex VI of the Commission Directive 76/768/EEC, and the improving directives code 2003/15/EC, 2007/17 EC, and 2007/22/EC which stated that the authorized and traditional preservatives are permitted to use in cosmetic preparations. Therefore, the word "self-preserving cosmetics" is more suitable for these products.

In self-preserving preparations, traditional preservatives have been replaced by other cosmetic ingredients that exert antimicrobial activity. Substances which have been used in self-preserving preparations are caprylyl alcohol, fatty acids, and their monoesters such as glyceryl monoester of caprylic, capric, and lauric acid, ethylhexylglycerin, chelating agents such as citric acid, lactic acid, and ethylenediaminetetraacetic acid, phenolic antioxidants, plant extracts, essential oils, and fragrance ingredients [1].

Plants are the best source of cosmeceutical compounds with antiaging and ultraviolet-A photoprotective properties. Indian herbs are used for the formulation of herbal cosmetics and sanitary products such as shampoo [5,6]. Phenethyl alcohol or phenylethyl alcohol (PEA) is naturally occurring aromatic compound that found in various flowers including rose, hyacinth, neroli, ylang-ylang, geranium, and champaca [7]. PEA is slightly soluble in water but miscible with alcohol and ether. PEA is commonly used in flavor and fragrance industries because of its flowery odor. The bacteriostatic activity of PEA was primarily reported by Lilley and Brewer [8], later studies revealed that PEA inhibits the DNA synthesis in Escherichia coli but not RNA and protein synthesis [9]. The bacteriostatic activity of PEA depends on the ability to impair the permeability of bacterial cell wall and increase the efflux rate of cellular potassium through the energydependent potassium pump [10]. In addition, some strains of E. coli were sensitive to PEA [11]. PEA is one of the major phytochemicals, responsible for medicinal property of the plant, present in the leaves of Rhododendron campanulatum [12]. The bioactivities, less irritability, and safety profiles evidenced that PEA may be a strong candidate in cosmetic preparations. The present study explored the use of PEA as a preservative in the standard emulsion, cleansing, and hair conditioner preparations. The microbial growth rate and other factors influencing 
the conservative nature of PEA such as $\mathrm{pH}$ and concentration have also been studied.

\section{METHODS}

\section{Chemicals and reagents}

The analytical grade of Eumulgin B3 (ceteareth-30), mineral oil, stearic acid, cetyl alcohol, glyceryl monostearate, glycerin, sodium lauryl ether sulfate, triethanolamine, citric acid, sodium chloride, and Tween 80 (polyoxyethylene sorbitan monooleate) was purchased from a local dealer in Thailand. Sabouraud dextrose agar, Pseudomonas agar F, and Pseudomonas agar P were purchased from Difco (DT, USA). Plate count agar (PCA), potato dextrose agar (PDA), tryptic soy broth and agar, and mannitol salt agar base were purchased from Merck (Darmstadt, Germany). The egg yolk emulsion was purchased from Oxoid (Thermo Scientific, UK). Cooked meat medium was purchased from BD (Becton Dickinson, USA). PEA was purchased from Tokyo Chemical Industry (Tokyo, Japan).

\section{Antimicrobial activity}

The antimicrobial activity of PEA was assessed by modified agar well plate assay method [13]. Briefly, bacterial strains of E. coli ATCC 25922, Staphylococcus aureus ATCC 25923, and Pseudomonas aeruginosa ATCC 27853 were cultured on tryptic soy agar. Candida albicans ATCC 90028 and Aspergillus spp. were cultured on sabouraud dextrose agar. The bacteria, yeast, and mold were diluted to $10^{8}$ and $10^{6} \mathrm{CFU} / \mathrm{ml}$ by comparing the turbidity with McFarland No. 0.5 , respectively, and were adjusted to a final concentration of $10^{6} \mathrm{CFU} / \mathrm{ml}$. A $1 \mathrm{ml}$ of diluted test culture was added to $10 \mathrm{ml}$ of their respective culture medium and poured on Petri plates to form the bottom layer. Then, the aluminum rings were placed on the medium, and again $10 \mathrm{ml}$ of the medium was poured on the top of the aluminum rings. After solidification of the medium, rings were removed carefully to create agar wells.

PEA at different concentrations or gentamicin $(15 \mathrm{mg} / \mathrm{ml})$ was poured in the agar well later served as a positive control for bacteria; amphotericin B $(15 \mathrm{mg} / \mathrm{ml})$ was used as a positive control for yeast and mold. The antimicrobial activity was observed and scored based on the following criteria. Strong inhibition - clear zone of more than $6 \mathrm{~mm} \mathrm{(+++),} \mathrm{moderate} \mathrm{inhibition} \mathrm{-} \mathrm{clear} \mathrm{zone} \mathrm{of} \mathrm{3-6} \mathrm{mm} \mathrm{(++),} \mathrm{and} \mathrm{weak}$ inhibition $-<3 \mathrm{~mm}$ sized clear zone (+). The antimicrobial activities of PEA-containing cosmetic formulations were also assessed by agar well diffusion method.

\section{Preparation of emulsion}

The test emulsion was composed of eumulgin B3 (2\% w/w), mineral oil $(5 \% \mathrm{w} / \mathrm{w})$, stearic acid $(3.5 \% \mathrm{w} / \mathrm{w})$, cetyl alcohol $(1.5 \% \mathrm{w} / \mathrm{w})$, and glyceryl monostearate $(2 \% \mathrm{w} / \mathrm{w})$ as an oil phase and glycerine $(2 \% \mathrm{w} / \mathrm{w})$, triethanolamine $(1.5 \% \mathrm{w} / \mathrm{w})$, and purified water as a water phase. The oil phase and water phase were heated separately at $85^{\circ} \mathrm{C}$; then, the oil phase was slowly incorporated into the water phase with continuous agitation. Thoroughly mixed emulsion was left at room temperature (RT) for cooling.

\section{Preparation of cleansing solution}

The cleansing solution was composed of sodium lauryl ether sulfate $(30 \% \mathrm{w} / \mathrm{w})$, sodium chloride $(2 \% \mathrm{w} / \mathrm{w})$, and purified water. Initially, the surfactant was softened in the water, and sodium chloride was gradually added.

\section{Preparation of hair conditioner}

The hair conditioner was prepared with eumulgin B3 (5\% w/w), mineral oil $(5 \% \mathrm{w} / \mathrm{w})$, glyceryl monostearate $(5 \% \mathrm{w} / \mathrm{w})$, cetyl alcohol $(3 \% \mathrm{w} / \mathrm{w})$, and stearic acid $(3 \% \mathrm{w} / \mathrm{w})$ as oil phase and propylene glycol $(3 \% \mathrm{w} / \mathrm{w})$, tween $80(2 \% \mathrm{w} / \mathrm{w})$, triethanolamine $(0.5 \% \mathrm{w} / \mathrm{w})$, and purified water as a water phase. Similar to emulsion preparation, the oil phase and water phase were heated separately at $85^{\circ} \mathrm{C}$, and then the oil phase was slowly incorporated into the water phase with continuous agitation. Thoroughly mixed emulsion was left at RT for cooling.

\section{Incorporation of PEA into preparations}

The influence of the concentration of PEA and $\mathrm{pH}$ of the cosmetic preparations was assessed. Thus, the prepared test products (emulsion, cleansing solution, and conditioner) were divided into 12 equal parts. The conservative nature of PEA was studied with four different concentrations $(0 \%, 0.3 \%, 1.0 \%$, and $2.5 \% \mathrm{w} / \mathrm{w})$ at three different $\mathrm{pH}$ $(4,6$, and 8$)$ in cosmetic products. Citric acid and triethanolamine were used to reduce and increase the $\mathrm{pH}$, respectively.

\section{Physical assessment of tested preparations}

Physical parameters such as physical appearance, color, and odor were assessed by organoleptic techniques, and $\mathrm{pH}$ of the samples was tested by the $\mathrm{pH}$ meter. The stability of each preparation was evaluated by heating-cooling cycle. Briefly, preparations were kept at $4^{\circ} \mathrm{C}$ for $48 \mathrm{hrs}$ then immediately transferred to $45^{\circ} \mathrm{C}$ for $48 \mathrm{~h}$ for 6 consecutive cycles.

\section{Quantification of microbial load}

Microbiological assessments of the formulations were tested based on the method of the US Food and Drug Administration: Bacteriological Analytical Manual: Microbiological Methods for cosmetics, and microbial standard suggested in the Thai Industrial Standard Institute (code: 152-2539; Cosmetics: General specification). The criteria from community product standard No. 92/2546 and 93/2546 were used for shampoo and hair conditioner, respectively. Total bacterial count was assessed by pour plate technique using PCA and PDA for bacteria, yeasts, and mold, respectively. The plates were incubated at $37^{\circ} \mathrm{C}$ for $24 \mathrm{~h}$ and $30^{\circ} \mathrm{C}$ for 3 days for bacteria, yeasts, and mold, respectively.

The samples $(0.1 \mathrm{ml})$ were cultured in mannitol salt egg yolk agar at $35-37^{\circ} \mathrm{C}$ for 2 days to check $S$. aureus content. The suspected colonies were further tested for coagulase activity to confirm $S$. aureus. The test samples ( $0.1 \mathrm{ml})$ were cultured in Pseudomonas agar F and Pseudomonas agar $\mathrm{P}$, cooked meat medium, and sabouraud dextrose agar at $35-37^{\circ} \mathrm{C}$ for 2-3 days to check P. aeruginosa, Clostridium spp., and C. albicans content, respectively.

\section{Statistical analysis}

All the values were symbolized as mean \pm standard deviation. Data were analyzed using SPSS 17.0 (2009 SPSS Inc., Chicago, IL, USA) by analysis of one-way analysis of variance. Differences were considered significant at $\mathrm{p}<0.05$.

\section{RESULTS AND DISCUSSION}

\section{Antimicrobial activity of PEA}

Antimicrobial activity of PEA was assessed against representative bacterial and fungal pathogens, namely, E. coli, S. aureus, P. aeruginosa, and $C$. albicans. The results were compared with known antibacterial agent gentamicin and antifungal agent amphotericin B. Gentamicin $(15 \mathrm{mg} / \mathrm{ml})$ showed a clear bacterial zone of $24 \pm 1,20 \pm 1$, and $20 \pm 2$ against E. coli, S. aureus, and P. aeruginosa, respectively. Whereas, absolute PEA showed similar or more antibacterial activity against tested pathogens compared to gentamicin. We have tested the antimicrobial activity of PEA at three different concentrations $(0.3 \%$, $1.0 \%$, and $2.5 \%$ ). About $2.5 \%$ of PEA exhibited a clear bacterial zone of $15 \pm 1,22 \pm 1$, and $19 \pm 1 \mathrm{~mm}$ against E. coli, S. aureus, and P. aeruginosa, respectively. The result indicates that $2.5 \%$ of PEA exhibited the antibacterial activity almost equal to $15 \mathrm{mg} / \mathrm{ml}$ of gentamicin, except against E. coli. Even though, $1.0 \%$ of PEA exhibited anti-S. aureus activity (Table 1).

All the tested PEA concentrations $(0.3 \%, 1.0 \%$, and $2.5 \%)$ showed strong anti-C. albicans activity $(22 \pm 1,24 \pm 1$, and $25 \pm 1 \mathrm{~mm}$, respectively), whereas absolute PEA and amphotericin $B(15 \mathrm{mg} / \mathrm{ml})$ showed $28 \pm 1$ and $22 \pm 1 \mathrm{~mm}$ of clear zone against $C$. albicans, respectively. The results suggested that the least concentration of PEA $(0.3 \%)$ was active against C. albicans when compared to the standard antifungal amphotericin $\mathrm{B}$ (Table 1). 
Physical property of cosmetic preparations

Since preservative can impair the physical characteristic of cosmetic preparations, we have assessed the physical properties of test cosmetic products. The model cosmetic formulations (emulsion, cleansing solution, and conditioner) were prepared with three different concentrations of PEA $(0.3 \%, 1.0 \%$, and $2.5 \%)$ at three different $\mathrm{pH}$ $(4,6$, and 8$)$ (Tables 2-4). The physical properties of tested emulsion were shown in Table 2 . All of the emulsions were homogenously milky lotion, opalescent liquid with fainted waxy to flowery odor. The odor changes due to the concentration of PEA used in the formula. The results implied that the addition of PEA does not show any adverse impact on the physical properties of the emulsion, and also the different $\mathrm{pH}$ values have not made any sense in this context (Table 2).

The physical properties of the cleansing solutions were shown in Table 3. All the cleansing formulas appeared as clear and clear viscous liquid with fainted to floral odor with numerous foam formation. The addition of $2.5 \%$ of PEA reduces the foam formation. The $\mathrm{pH}$ of the preparation influences the viscosity of the cleansing solution, in detail, the preparations with $\mathrm{pH} 4$ appeared as a clear liquid (less viscous), irrespective of the concentration of PEA (Table 3).
The conditioner formulations appeared as a homogenous white lotion, with collapsed odor of the chemicals used. The preparations with $1.0 \%$ and $2.5 \%$ of PEA smelled like mild floral to floral, whereas other formulas were in waxy odor. The $\mathrm{pH}$ and concentration of PEA have not severely affected the physical parameter of the conditioner (Table 4). All the cosmetic formulations passed the stability test after 1 month of storage duration. It means that the physical integrity, odor, and general acceptability of the product were not affected by the addition of PEA (Tables 2-4).

The results revealed that the addition of PEA did not interfere the physical properties of tested emulsion and conditioner, but the high amount of PEA $(2.5 \% \mathrm{w} / \mathrm{w})$ in the cleansing formulations decreases the foam formation. The defoaming effect is due to the nature of alcohol, thereby, alcohol reduces the foam effect of surfactant solution. Rosy and flowery odor of PEA gave a pleasant fragrance to the formulations, thus no need of adding additional other synthetic fragrances.

Microbiological assessments

The microbial assessments of the tested formulations were conducted under the criterion of the Thai Industrial Standard (code 152-2539;

Table 1: Antimicrobial activity of PEA against selected pathogens

\begin{tabular}{llll}
\hline \multirow{2}{*}{ Tested compound } & \multicolumn{2}{l}{ Mean \pm SD diameter of inhibition zone $(\mathbf{m m})$} & Pseudomonas aeruginosa \\
\cline { 2 - 4 } & Escherichia coli & Staphylococcus aureus & $20 \pm 1$ \\
\hline Gentamicin & $24 \pm 1$ & $20 \pm 2$ & $\mathrm{NA}$ \\
Amphotericin B & $\mathrm{NA}$ & $\mathrm{NA}$ & $22 \pm 1^{\mathrm{d}}$ \\
Absolute PEA & $25 \pm 1^{\mathrm{d}}$ & $22 \pm 1^{\mathrm{d}}$ & $11 \pm 1^{\mathrm{a} *}$ \\
$0.3 \%$ PEA & $8 \pm 0.5^{\mathrm{a} *}$ & $18 \pm 1^{\mathrm{a} *}$ & $15 \pm 1^{\mathrm{b} *}$ \\
$1.0 \%$ PEA & $12 \pm 1^{\mathrm{b} *}$ & $22 \pm 2^{\mathrm{b}}$ & $19 \pm 2^{\mathrm{c}}$ \\
$2.5 \%$ PEA & $15 \pm 1^{\mathrm{c} *}$ & $25 \pm 1^{\mathrm{c} *}$ & $22 \pm 2^{\mathrm{b} *}$ \\
\hline
\end{tabular}

NA: Not applicable. a-dRepresent the difference between the groups. ${ }^{\text {ad }}$ Represent the significant difference between antibiotic standards and PEA ( $\left.<<0.05\right)$. PEA: Phenethyl alcohol, SD: Standard deviation

Table 2: Physical properties of emulsions with different $\mathrm{pH}$ and concentration of PEA

\begin{tabular}{|c|c|c|c|c|c|}
\hline Formulation & pH & Concentration of PEA $(\% \mathrm{w} / \mathrm{w})$ & Physical appearance & Odor & Stability \\
\hline Emulsion 1 & 4 & 0 & Opalescent liquid & Waxy & Pass \\
\hline Emulsion 2 & 6 & 0 & Opalescent liquid & Waxy & Pass \\
\hline Emulsion 3 & 8 & 0 & Opalescent liquid & Waxy & Pass \\
\hline Emulsion 4 & 4 & 0.3 & Opalescent liquid & Waxy & Pass \\
\hline Emulsion 5 & 6 & 0.3 & Opalescent liquid & Waxy & Pass \\
\hline Emulsion 6 & 8 & 0.3 & Opalescent liquid & Waxy & Pass \\
\hline Emulsion 7 & 4 & 1.0 & Opalescent liquid & Mild floral & Pass \\
\hline Emulsion 9 & 8 & 1.0 & Opalescent liquid & Mild floral & Pass \\
\hline Emulsion 10 & 4 & 2.5 & Opalescent liquid & Floral & Pass \\
\hline Emulsion 11 & 6 & 2.5 & Opalescent liquid & Floral & Pass \\
\hline Emulsion 12 & 8 & 2.5 & Opalescent liquid & Floral & Pass \\
\hline
\end{tabular}

PEA: Phenethyl alcohol

Table 3: Physical properties of cleansing solutions with different pH and concentration of PEA

\begin{tabular}{|c|c|c|c|c|c|c|}
\hline Formulation & pH & Concentration of PEA ( $\% \mathrm{w} / \mathrm{w})$ & Physical appearance & Foam & Odor & Stability \\
\hline Cleansing 1 & 4 & 0 & Clear liquid & Numerously & Fainted & Pass \\
\hline Cleansing 2 & 6 & 0 & Clear viscous liquid & Numerously & Fainted & Pass \\
\hline Cleansing 3 & 8 & 0 & Clear viscous liquid & Numerously & Fainted & Pass \\
\hline Cleansing 4 & 4 & 0.3 & Clear liquid & Numerously & None & Pass \\
\hline Cleansing 5 & 6 & 0.3 & Clear viscous liquid & Numerously & None & Pass \\
\hline Cleansing 6 & 8 & 0.3 & Clear viscous liquid & Numerously & None & Pass \\
\hline Cleansing 7 & 4 & 1.0 & Clear liquid & Numerously & Mild floral & Pass \\
\hline Cleansing 9 & 8 & 1.0 & Clear viscous liquid & Numerously & Mild floral & Pass \\
\hline Cleansing 10 & 4 & 2.5 & Clear liquid & Sparingly & Floral & Pass \\
\hline Cleansing 11 & 6 & 2.5 & Clear viscous liquid & Sparingly & Floral & Pass \\
\hline Cleansing 12 & 8 & 2.5 & Clear viscous liquid & Sparingly & Floral & Pass \\
\hline
\end{tabular}

PEA: Phenethyl alcohol 
"Cosmetics: General specification") and test for the presence of contaminating microbes such as $S$. aureus, $P$. aeruginosa, C. albicans, and Clostridium spp. The microbiological assessment data were represented in Table 5. The preparations without the addition of PEA were more vulnerable to microbial spoilage after 1 month of storage at RT. The emulsion and cleansing formulations were more prone to bacterial growth, whereas conditioner formulas were enriched with mold contamination after storage at RT for 1 month. Yeast growth was recorded in all the formulations with a low concentration of PEA $(0.3 \%)$ and $\mathrm{pH} 8$, whereas no yeast growth was observed in the preparations with the $\mathrm{pH}$ of 4 and 6 . While the conditioner formulas at all tested $\mathrm{pH}$ ranges $(4,6$, and 8$)$ with $1.0 \%$ of PEA displayed the microbial growth, surprisingly other formulations were found to be free from contamination (Table 5).

S. aureus was found to be present in all the formulations without PEA and also in conditioner formulas with $1.0 \%$ of PEA at all pH ranges. Whereas, P. aeruginosa, C. albicans, and Clostridium spp. were not found in any of the tested formulations. The results suggested that the use of PEA in all tested products was conformed to the Thai Industrial Standard. Moreover, the results suggested that the activity of PEA is possibly $\mathrm{pH}$ dependent. Even though, the low concentration of PEA exhibited better activity at $\mathrm{pH} 8$ while not observed at low $\mathrm{pH}$ (Table 5).
. However, high content of PEA in cosmetic formulations was not $\mathrm{pH}$ dependent, especially, in antimicrobial activity. In emulsion and cleansing solution, the minimal required concentration of PEA was $1.0 \%$ at any tested $\mathrm{pH}$ range, but in the conditioner formulations, the minimum PEA concentration for better preservation was $2.5 \%$. The conditioners with $1.0 \%$ of PEA may facilitate the microbial growth.

\section{Antimicrobial activity of cosmetic formulas}

The antimicrobial activity of cosmetic preparations with PEA was shown in Table 6. There was no antimicrobial activity observed in emulsions and conditioners. All tested cleansing formulas displayed antimicrobial activity against E. coli, S. aureus, P. aeruginosa, C. albicans, and Aspergillus spp. (Table 6). The antimicrobial property of the cleansing formulations was possibly due to the presence of a surfactant, which may enhance the antimicrobial property of PEA, and the results were more comparable with gentamicin and amphotericin B (Table 6).

To the best of our knowledge, there is no detailed scientific report on the antimicrobial property of PEA in cosmetic products. PEA causes a quick and reversible interruption in the permeability of bacterial cells, which affect the function of intracellular organs and also inhibit the DNA synthesis [8,10,14-16]. Recently, Fang et al. [17] reported about the antimicrobial activity of combination of caprylyl glycol, PEA, and

Table 4: Physical properties of hair conditioners with different $\mathrm{pH}$ and concentration of PEA

\begin{tabular}{|c|c|c|c|c|c|}
\hline Formulation & pH & Concentration of PEA $(\% w / w)$ & Physical appearance & Odor & Stability \\
\hline Conditioner 1 & 4 & 0 & Opalescent liquid & Waxy & Pass \\
\hline Conditioner 2 & 6 & 0 & Opalescent liquid & Waxy & Pass \\
\hline Conditioner 4 & 4 & 0.3 & Opalescent liquid & Waxy & Pass \\
\hline Conditioner 5 & 6 & 0.3 & Opalescent liquid & Waxy & Pass \\
\hline Conditioner 6 & 8 & 0.3 & Opalescent liquid & Waxy & Pass \\
\hline Conditioner 7 & 4 & 1.0 & Opalescent liquid & Mild floral & Pass \\
\hline Conditioner 8 & 6 & 1.0 & Opalescent liquid & Mild floral & Pass \\
\hline Conditioner 9 & 8 & 1.0 & Opalescent liquid & Mild floral & Pass \\
\hline Conditioner 10 & 4 & 2.5 & Opalescent liquid & Floral & Pass \\
\hline Conditioner 11 & 6 & 2.5 & Opalescent liquid & Floral & Pass \\
\hline Conditioner 12 & 8 & 2.5 & Opalescent liquid & Floral & Pass \\
\hline
\end{tabular}

PEA: Phenethyl alcohol

Table 5: Microbiological assessment of emulsions, cleansing solutions, and conditioners after being kept at RT for 1 month

\begin{tabular}{|c|c|c|c|c|c|}
\hline \multirow[t]{2}{*}{ Formulation } & \multicolumn{5}{|c|}{ Microbial count (CFU/g of sample) } \\
\hline & Total colony count & Staphylococcus aureus & $\begin{array}{l}\text { Pseudomonas } \\
\text { aeruginosa }\end{array}$ & Candida albicans & Clostridium spp. \\
\hline Emulsion 1 & Bacteria $3.65 \times 10^{3}$ & $1.31 \times 10^{2}$ & - & - & - \\
\hline Emulsion 2 & Bacteria $2.24 \times 10^{3}$ & $1.42 \times 10^{2}$ & - & - & - \\
\hline Emulsion 3 & Bacteria $1.67 \times 10^{3}$ & $1.61 \times 10^{2}$ & - & - & - \\
\hline Emulsion 4 & - & - & - & - & - \\
\hline Emulsion 6 & Yeast $4.67 \times 10^{3}$ & - & - & - & - \\
\hline Emulsion 7-12 & - & - & - & - & - \\
\hline Cleansing 1 & Bacteria $3.22 \times 10^{4}$ & $1.31 \times 10^{2}$ & - & - & - \\
\hline Cleansing 2 & Bacteria $2.65 \times 10^{4}$ & $1.22 \times 10^{2}$ & - & - & - \\
\hline Cleansing 3 & Bacteria $3.01 \times 10^{4}$ & $1.46 \times 10^{2}$ & - & - & - \\
\hline Cleansing 4, 5 & - & - & - & - & - \\
\hline Cleansing 6 & Yeast $2.23 \times 10^{2}$ & - & - & - & - \\
\hline Cleansing 7-12 & - & - & - & - & - \\
\hline Conditioner 2 & Mold $2.26 \times 10^{3}$ & $1.06 \times 10^{2}$ & - & - & - \\
\hline Conditioner 3 & Mold $3.55 \times 10^{3}$ & 88 & - & - & - \\
\hline Conditioner 4, 5 & - & - & - & - & - \\
\hline Conditioner 6 & Yeast $3.57 \times 10^{2}$ & - & - & - & - \\
\hline Conditioner 7 & Yeast $1.44 \times 10^{2}$ & 84 & - & - & - \\
\hline Conditioner 8 & Bacteria $1.68 \times 10^{5}$ & $2.63 \times 10^{2}$ & - & - & - \\
\hline Conditioner 9 & Bacteria $2.53 \times 10^{5}$ & $4.12 \times 10^{2}$ & - & - & - \\
\hline Conditioner 10-12 & - & - & - & - & - \\
\hline
\end{tabular}

RT: Room temperature 
Table 6: Antimicrobial activities of each formulation assessed by agar plate assay

\begin{tabular}{|c|c|c|c|c|c|}
\hline \multirow[t]{2}{*}{ Formulation } & \multicolumn{5}{|c|}{ Diameter of inhibition zone (mm) } \\
\hline & Escherichia coli & Staphylococcus aureus & $\begin{array}{l}\text { Pseudomonas } \\
\text { aeruginosa }\end{array}$ & Candida albicans & Aspergillus spp. \\
\hline Gentamicin $15 \mu \mathrm{g} / \mu \mathrm{l}$ & 18 & 20 & 18 & NA & NA \\
\hline Amphotericin B $15 \mu \mathrm{g} / \mu \mathrm{l}$ & NA & NA & NA & 20 & 20 \\
\hline Emulsion 1-12 & - & - & - & - & - \\
\hline Cleansing 1-3 & - & - & - & - & - \\
\hline Cleansing 5 & 20 & 16 & 12 & 18 & 16 \\
\hline Cleansing 6 & 16 & 14 & 12 & 20 & 16 \\
\hline Cleansing 7 & 16 & 20 & 16 & 22 & 16 \\
\hline Cleansing 8 & 18 & 14 & 18 & 20 & 16 \\
\hline Cleansing 9 & 16 & 16 & 16 & 14 & 12 \\
\hline Cleansing 10 & 20 & 16 & 12 & 18 & 16 \\
\hline Cleansing 11 & 18 & 16 & 16 & 22 & 18 \\
\hline Cleansing 12 & 22 & 18 & 14 & 30 & 22 \\
\hline
\end{tabular}

NA: Not applicable

glyceryl caprylate with respect to the particle size of the emulsion, and they found that the antimicrobial ability of the mixture was improved progressively when the particle size of the emulsion increases from 100 to $900 \mathrm{~nm}$.

\section{CONCLUSION}

The present study revealed that the use of PEA in the model cosmetic formulations reduces the microbial growth during storage. The minimal required concentration of PEA for emulsion and the cleansing solutions was $1.0 \%$ while $2.5 \%$ for conditioner at $\mathrm{pH} 4-6$ to prevent the contamination during storage at RT. However, the extensive studies on the use of PEA in other cosmetic types such as solution, elixir, hydrogel, and lipid-based products are desirable. PEA is an active, potent substitute for traditional preservatives, which can be used in various cosmetic formulations to decrease the uses of traditional chemicals and to avoid the harmful effects.

\section{ACKNOWLEDGMENT}

All the authors thankfully acknowledge the Faculty of Pharmacy and Chiang Mai University, Chiang Mai for the necessary support. PK also would like to acknowledge the CMU Post-Doctoral Fellowship.

\section{REFERENCES}

1. Varvaresou A, Papageorgiou S, Tsirivas E, Protopapa E, Kintziou H, Kefala V, et al. Self-preserving cosmetics. Int J Cosmet Sci 2009;31(3):163-75.

2. Siquet F, Devleeschouwer MJ. Antibacterial agents and preservatives. In: Andre OB, Marc P, Howard IM, editors. Handbook of Cosmetic Science and Technology. USA: Marcel Dekker; 2001. p. 245-51.

3. Darbre PD, Aljarrah A, Miller WR, Coldham NG, Sauer MJ, Pope GS. Concentrations of parabens in human breast tumours. J Appl Toxicol 2004:24(1):5-13

4. Steinberg DC. Back to basics: So you want to preserve your emulsion. J Cosmet Sci 2007:58(5):574-5.
5. Rojas J, Londono C, Ciro Y. The health benefits of natural skin UV-A photoprotective compounds found in botanical sources. Int J Pharm Pharm Sci 2016;8(3):13-23.

6. Patel I, Talathi A. Use of traditional Indian herbs for the formulation of shampoo and their comparative analysis. Int J Pharm Pharm Sci 2016;8(3):28-32.

7. The Flavor and Fragrance High Production Volume Chemical Consortia. The Aromatic Consortium. Washington, DC: Test Plan for Phenethyl Alcohol; 2002

8. Lilley BD, Brewer JH. The selective antibacterial action of phenylethyl alcohol. J Am Pharm Assoc Am Pharm Assoc 1953;42(1):6-8.

9. Berrah G, Konetzka WA. Selective and reversible inhibition of the synthesis of bacterial deoxyribonucleic acid by phenethyl alcohol. J Bacteriol 1962;83:738-44.

10. Silver S, Wendt L. Mechanism of action of phenethyl alcohol: Breakdown of the cellular permeability barrier. J Bacteriol 1967;93(2):560-6.

11. Nakamura H. Phenethyl alcohol sensitivity in Escherichia coli. J Bacteriol 1967;93(3):1183-4

12. Painuli S, Rai N, Kumar N. GC-MS analysis of methanolic extract of leaves of Rhododendron campanulatum. Int J Pharm Pharm Sci 2015;7(12):299-303

13. Selvam TP, Sivakumar A, Prabhu PP. Design and synthesis of quinazoline carboxylates against gram-positive, gram-negative, fungal pathogenic strains, and Mycobacterium tuberculosis. J Pharm Bio Allied Sci 2014;6(4):278-84.

14. Masker WE, Eberle H. Effect of phenethyl alcohol on deoxyribonucleic acid-membrane association in Escherichia coli. $\mathrm{J}$ Bacteriol 1972;109(3):1170-4.

15. Fraud S, Rees EL, Mahenthiralingam E, Russell AD, Maillard JY. Aromatic alcohols and their effect on Gram-negative bacteria, cocci and mycobacteria. J Antimicrob Chemother 2003;51:1435-6.

16. Tieman DM, Loucas HM, Kim JY, Clark DG, Klee HJ. Tomato phenylacetaldehyde reductases catalyze the last step in the synthesis of the aroma volatile 2-phenylethanol. Phytochemistry 2007;68(21):2660-9.

17. Fang $\mathrm{B}, \mathrm{Yu}$ M, Zhang W, Wang F. A new alternative to cosmetics preservation and the effect of the particle size of the emulsion droplets on preservation efficacy. Int J Cosmet Sci 2016;38(5):496-503. 